Connecticut College

Digital Commons @ Connecticut College

1909

\title{
Love Thy Neighbor As Thyself
}

Will A. Heelan

E. S. S. Huntington

Seymour Furth

Follow this and additional works at: https://digitalcommons.conncoll.edu/sheetmusic

\section{Recommended Citation}

Heelan, Will A.; Huntington, E. S. S.; and Furth, Seymour, "Love Thy Neighbor As Thyself" (1909). Historic Sheet Music Collection. 1423.

https://digitalcommons.conncoll.edu/sheetmusic/1423

This Score is brought to you for free and open access by the Greer Music Library at Digital Commons @ Connecticut College. It has been accepted for inclusion in Historic Sheet Music Collection by an authorized administrator of Digital Commons @ Connecticut College. For more information, please contact bpancier@conncoll.edu.

The views expressed in this paper are solely those of the author. 

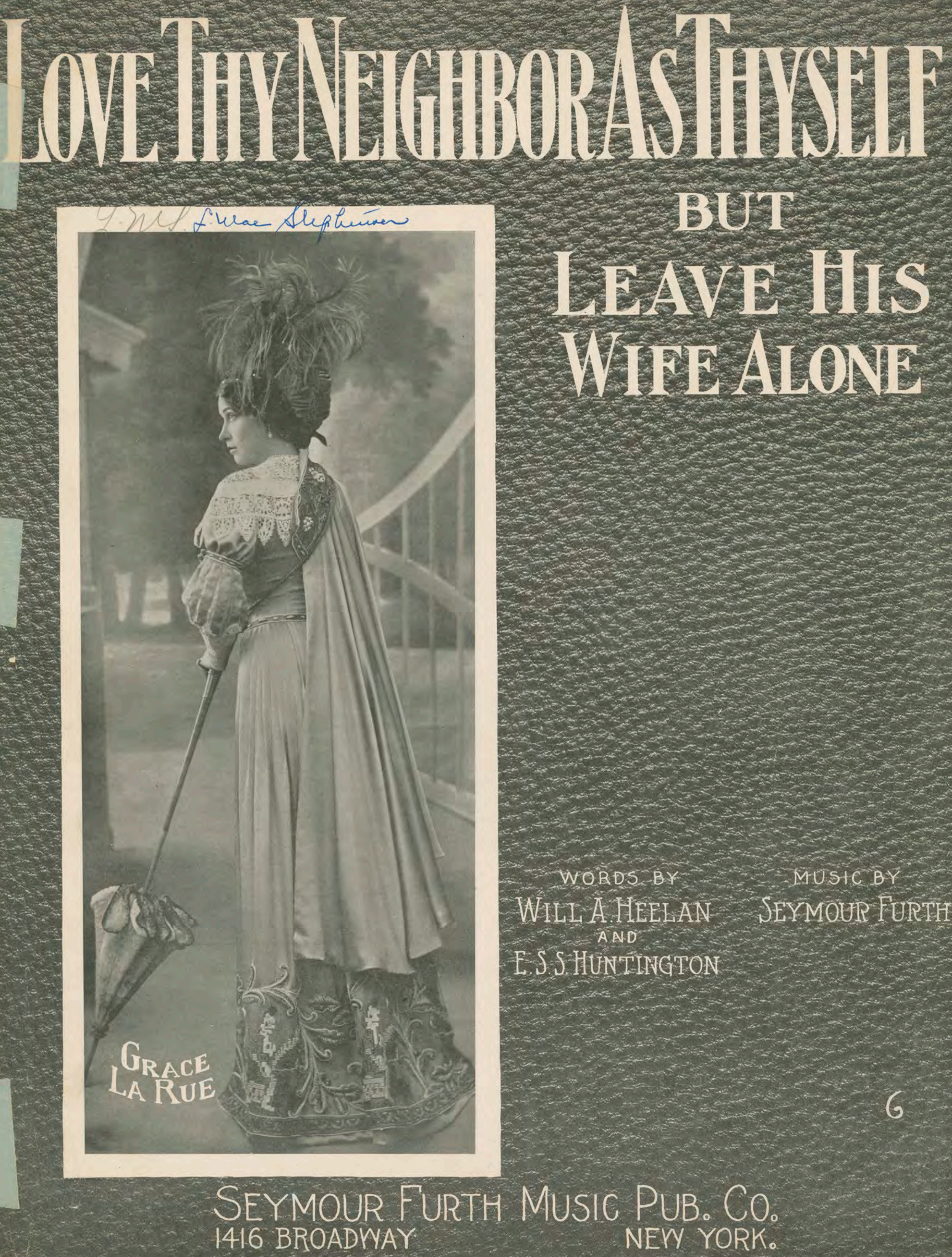

SEYMOUR FURTH MUSIC PUB. CO,
I416 BROADWAY
NEM YORK. 


\section{Try this over on your Piano.}

I Wonder If This Funny Little Boy Loves Me

Words by

GE0 W. DAY.
Music by

SEYMOUR FURTH.
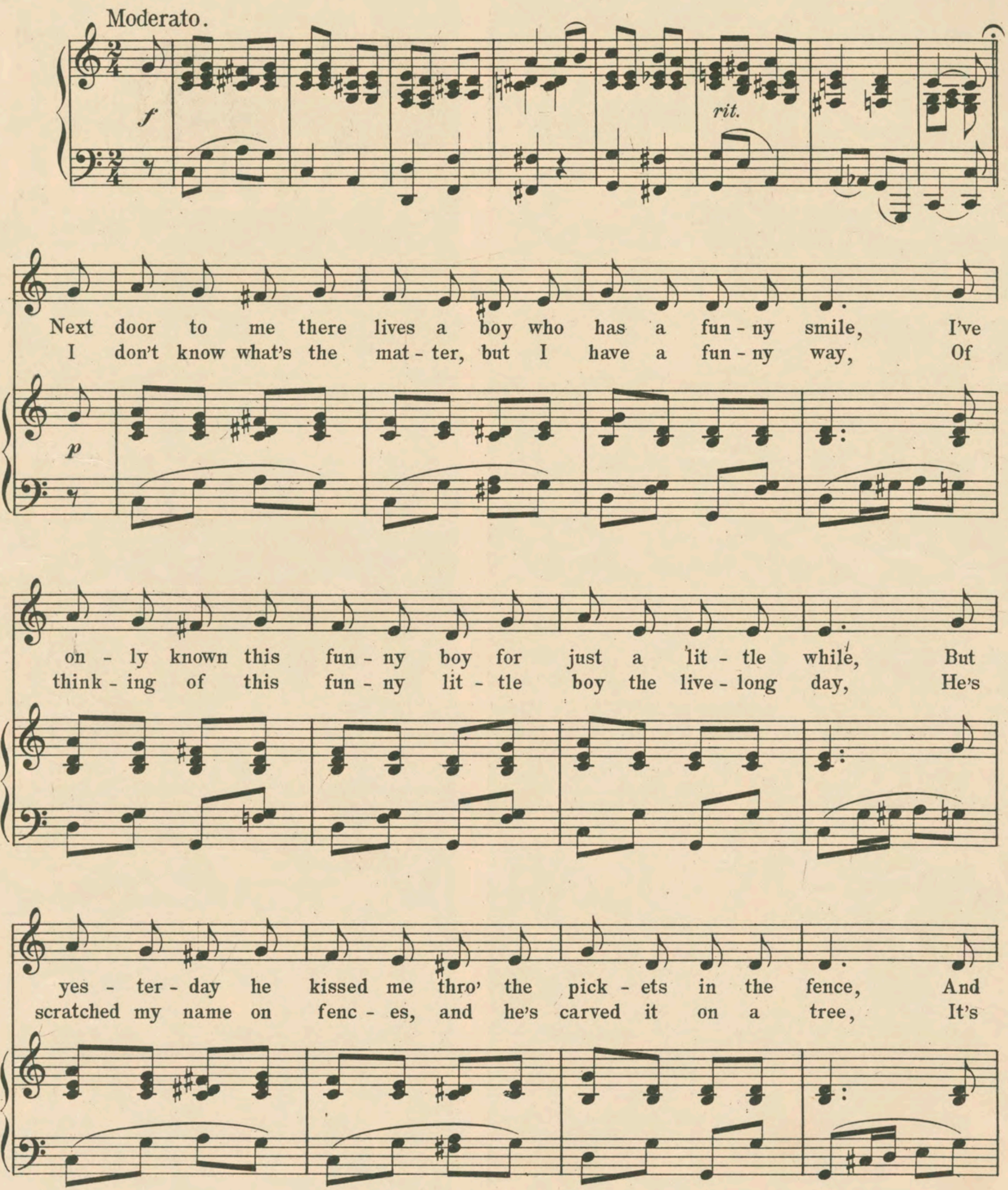

Copyright 1909 by The Seymour Furth Music Pub. Co. 1416 Broadway, N. Y. International Copyright Secured.

Complete Copies may be had where you bought this. 
Words by

\section{Love Thy Neighbor As Thyself, \\ But \\ Leave His Wife Alone.}

WILL A. HEELAN \&

E.S.S.HUNTINGTON.

Music by

SEYMOUR FURTH.
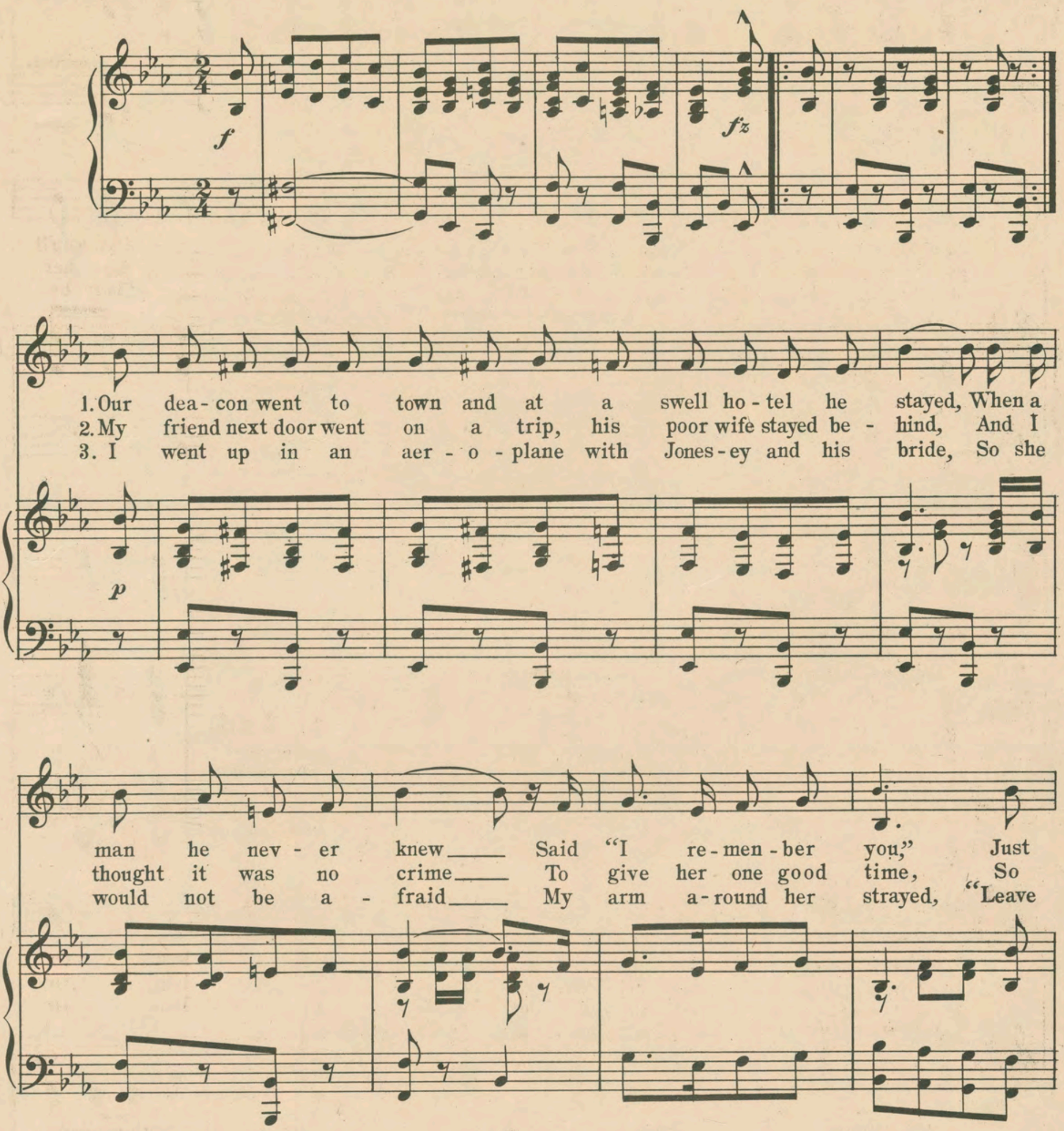

Copyright 1909 by The Seymour Furth Music Pub.Co. 1416 Broadway N.Y. International Copyright Secured. 

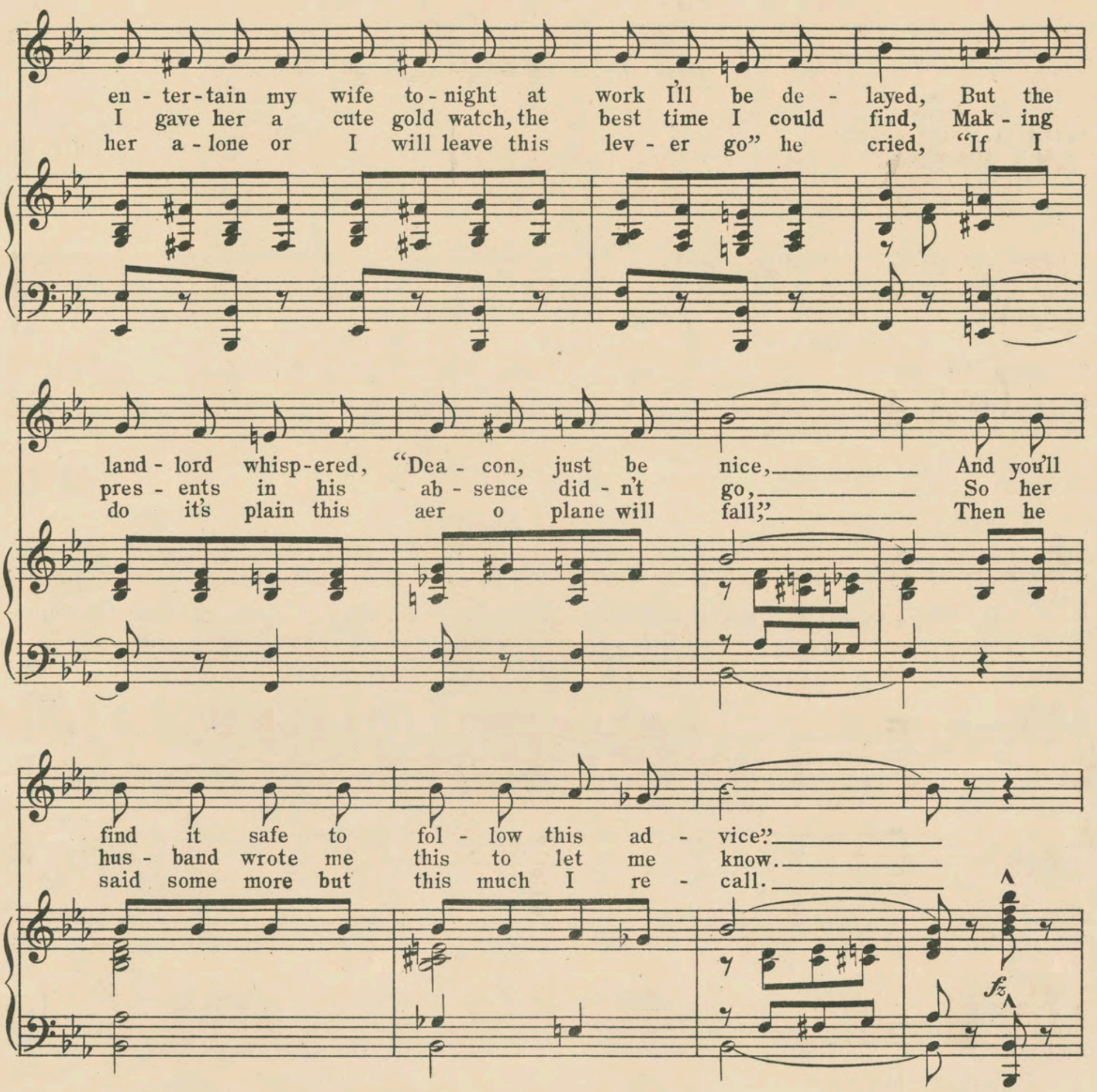

CHORUS. SIow.

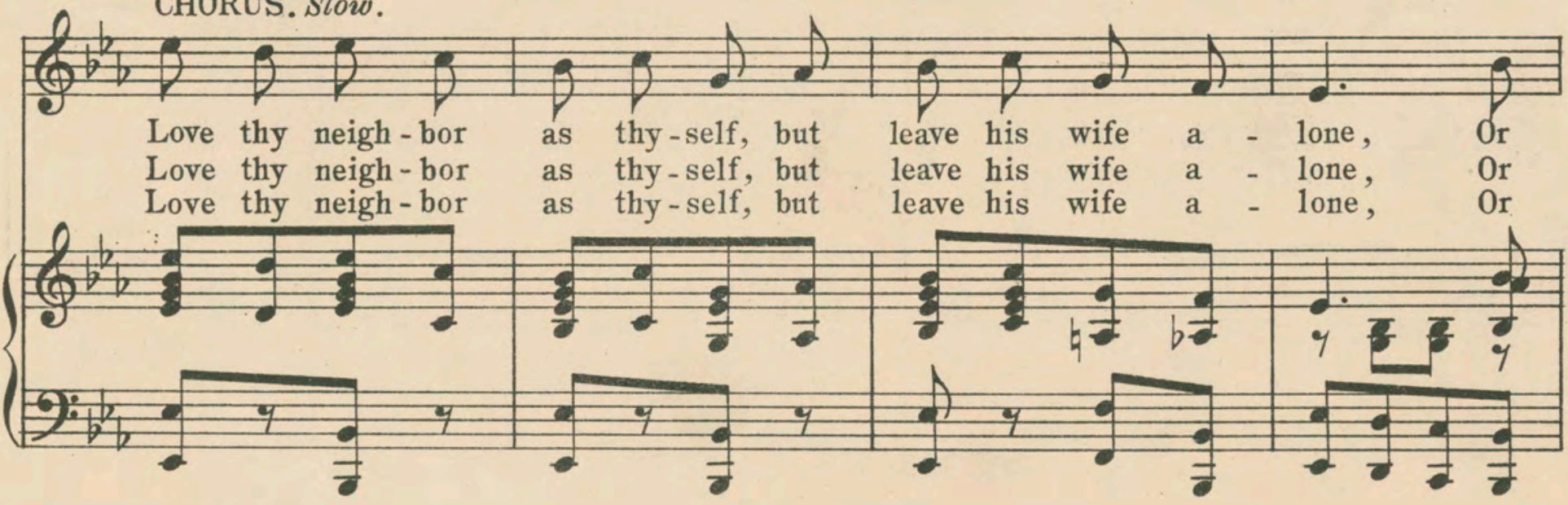

Love Thy Neighbor etc.3. 

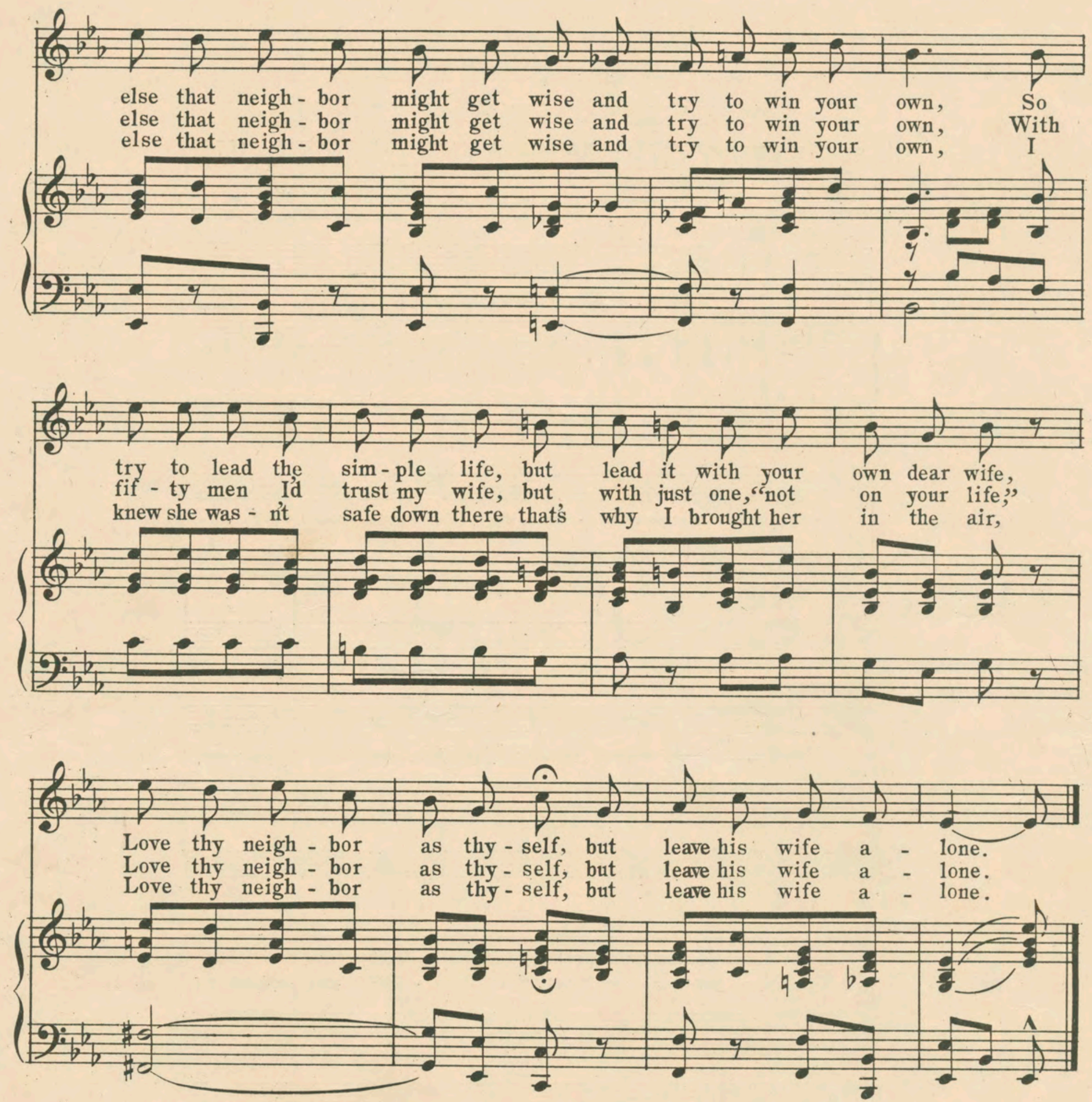

A male and female on a fence were singing in B Flat

It's an ordinary thing, to hear two young cats sing,

They sang the latest catalogue of catawauls at that

When another Tom yelled "What is this I see?

Quit my bride or there'll be some catastrophy:

CHORUS.

Love thy neighbor as thyself but leave his wife alone

Or else that neighbor might get wise and try to win your own

"I cantbring suit, I think it's tough

A back fence isn't grounds enough

Love thy neighbor as thyself but leave his wife alone.

Love Thy Neighbor etc.3. 
THIS SEASON'S SONG

THIS SEASON'S WALTZ SONG SUCCESS, "IF EVERY GIRL WAS A GIRL LIKE YOU."
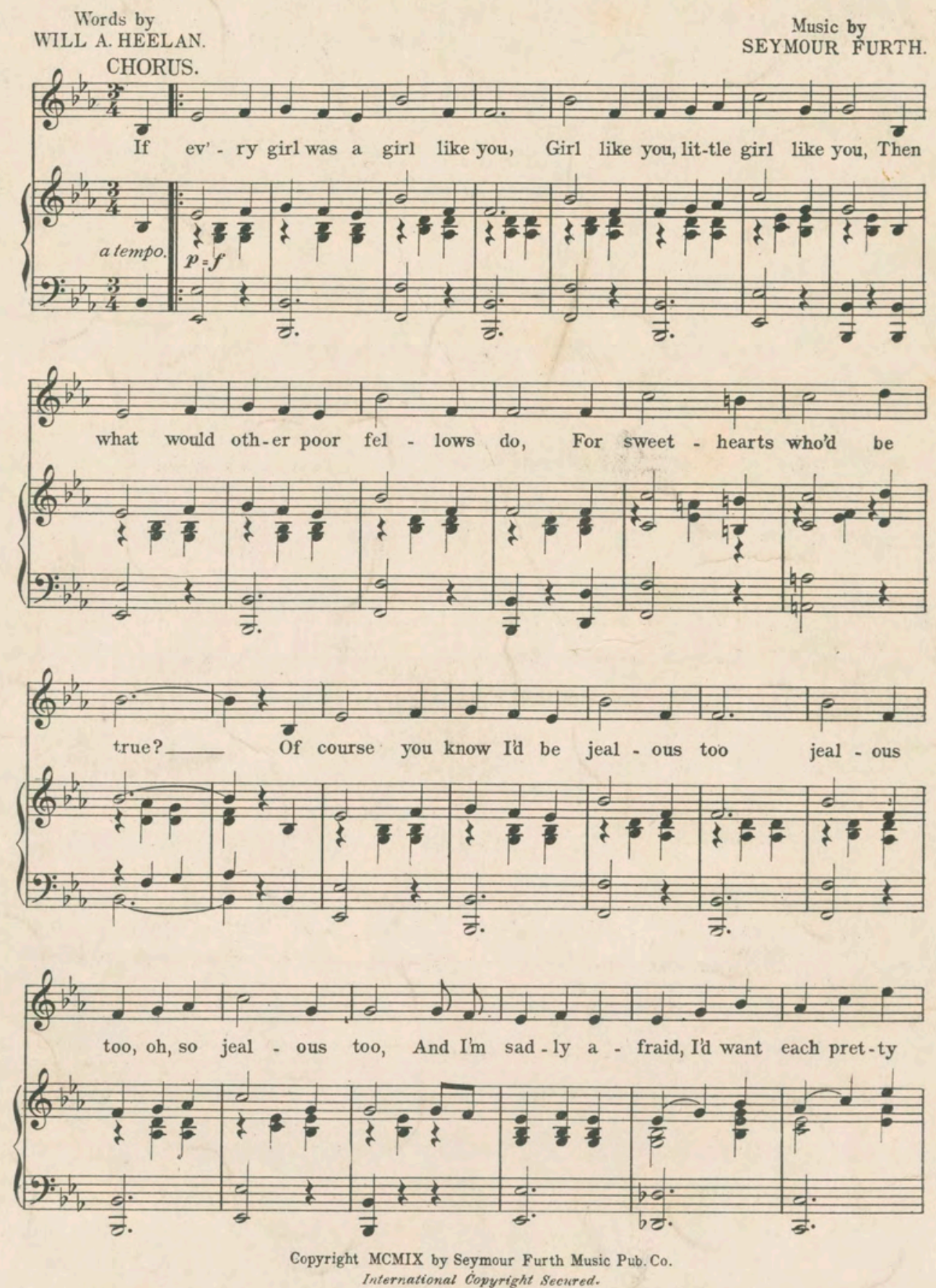

SOLD WHERE THEY SELL SONG HITS.

OR FROM

SEYMOUR FURTH MUSIC PUB CO.

1416 BROADWAY, N. Y. CITY. 